\title{
Approximation of Complex Harmonic Functions by Complex Harmonic Splines
}

\author{
By Han-Lin Chen and Tron Hvaring
}

\begin{abstract}
In this paper, a class of complex harmonic spline functions (C.H.S.) are defined on the unit disc $U$. We use the C.H.S. to approximate the complex harmonic function on $U$, showing that C.H.S. may be represented by elementary functions. If the maximum step tends to zero and the mesh ratio is bounded, then C.H.S. converge uniformly to the interpolated function $F$ on the closed disc $\bar{U}$. If the interpolated function $F$ is a conformal mapping, then the C.H.S. is a quasi-conformal mapping.
\end{abstract}

1. Introduction. This paper deals with the spline approximation of a complex harmonic function defined on the unit disc $U$.

Since an important subclass of complex harmonic functions is the class of analytic functions, we are naturally interested in the following problem: can we use the splines to approximate a conformal mapping? We shall explain the reasons for using splines for this purpose.

It is well known that any simply connected domain $D$ contained in a closed Riemann surface, whose boundary $\Gamma$ is a continuum, can be mapped conformally on $|Z|<1$.

In practice, the important thing is to construct the mapping function, but as we know, if the domain is arbitrarily prescribed, it is difficult to obtain the analytic expression of the mapping function. For this reason, mathematicians devote a lot of work to develop approximations to the mapping function.

Recently, L. Reichel used the Lagrange interpolation polynomials for equi-spaced points on the unit circle $\gamma$ to approximate the mapping function which maps the unit disc $U$ onto a simply connected domain $D[9, \mathrm{pp} .32-34]$. The data of function values were obtained by solving a system of integral equations derived from $D$. Gaier's theory [7]. The method used in that paper [9], combined with analytic techniques and Fast Fourier transformation, is effective in some cases.

We note, however, that in general, the Lagrange interpolation polynomials do not converge uniformly to the mapping function $F(Z)$ on the closed disc $\bar{U}$ [6].

Ahlberg, Nilson and Walsh [1], [2] used analytic splines. These functions can be proved to converge uniformly on a closed point set interior to $\gamma$, but not on the closed disc $\bar{U}$.

Instead of Lagrange interpolation polynomials and analytic splines, we used complex harmonic splines to approximate a complex harmonic function defined on $U$. The formulas and the error bounds were given in [3].

Received September 17, 1982; revised December 6, 1982 and February 8, 1983.

1980 Mathematics Subject Classification. Primary 30C30; Secondary 41 A 15. 
The complex harmonic splines (C.H.S.) have the following properties:

(i) If the maximum step $|\Delta|$ tends to zero, and the mesh ratio $R$ is bounded, then C.H.S. converge uniformly to the interpolated function $F(Z)$ on the closed disc $\bar{U}$.

(ii) C.H.S. may be represented by elementary functions.

(iii) If the interpolated function $F(Z)$ maps $U$ conformally onto a simply connected domain $D$ satisfying the Ljapunov condition (see after Remark 2 ) and $|\Delta|=\max _{j}\left|Z_{j}-Z_{j-1}\right|$ (the maximum step) is sufficiently small, then the C.H.S. maps $U$ onto a simply connected domain and this mapping is univalent, in fact, it is a quasi-conformal mapping [3].

In [3], we used complex pseudo-interpolation splines as the boundary function of C.H.S., and we stipulated that the approximated functions $F(Z)$ have absolutely continuous $n$th derivatives $(n \geqslant 2)$ on $\gamma$ (see [3, Theorems 1-4]), where the pseudointerpolation splines are piecewise polynomials of degree $n$.

In this paper, we use the complex cubic interpolation spline function as the boundary function of C.H.S. Under a much weaker condition we can prove that (i) and (ii) are still valid, and we obtain the error bound in an explicit form (Theorems 1,2).

Under a weaker condition, we prove that (iii) is also valid (Theorems 3,4 ).

In the last paragraph of this paper we give formulas for the calculation of the C.H.S. and a program which implements the C.H.S. in practice:

If $\zeta=\zeta(t), 0 \leqslant t<2$, is the parametric representation of the Jordan curve $\Gamma$, then the following program is performed.

Input: $\zeta=\zeta(t)$. Output: The graph of C.H.S., where C.H.S. is the approximation of the conformal mapping $F(Z)$ which maps $U$ onto a simply connected domain $D$.

To save space, we delete the program and the graphs; they may be found in [5].

2. C.H.S. Approximation. A real function $u(x, y)$ belonging to $C^{2}(D)$ is said to be harmonic in $D$ if it satisfies the Laplace equation $\Delta u=0$.

If $u_{j}(j=1, \ldots, n)$ are harmonic functions in $D$, and $\alpha_{j}(j=1, \ldots, n)$ are complex numbers, then the function $u=\sum_{1}^{n} \alpha_{j} u$, also belongs to $C^{2}(D)$ and satisfies the Laplace equation, but $u$ is a complex-valued function; we call it a complex harmonic function and denote by $H(D)$ the family of all such functions defined on $D$.

A nonconstant complex harmonic function $u(Z)$ cannot have a maximal absolute value in its domain of definition. Consequently, the maximal value $|u(Z)|$ on a closed set $E$ is attained on the boundary of $E$.

$H(D)$ contains a wide class of functions, for instance, the function $P(Z)$ defined by the Poisson formula

$$
P(Z)=\frac{1}{2 \pi} \int_{0}^{2 \pi} w\left(e^{i \theta}\right) \operatorname{Re}\left(\frac{e^{i \theta}+Z}{e^{i \theta}-Z}\right) d \theta, \quad Z \in U,
$$

where $w(\zeta)$ is any complex continuous function defined on $\gamma, \gamma$ is the boundary of $U$, and $U$ the unit disc; evidently, $P(Z) \in H(U)$.

Let $\Delta=\left\{Z_{1}, \ldots, Z_{N}\right\}$ be points on $\gamma$ arranged in counterclockwise order, separating $\gamma$ into $\gamma_{j}(j=\overline{1, N})$ with $\gamma_{j}$ being the arc from $Z_{j}$ to $Z_{j+1}\left(Z_{N+1}=Z_{1}\right)$.

We stipulate: $\left|\widehat{Z}_{j} Z_{j+1}\right|=\left|\gamma_{j}\right| \leqslant \pi(j=\overline{1, N})$. 
Given complex data $Y_{1}, \ldots, Y_{N}$, there exists one and only one complex cubic spline function $S(Z)$ with nodes $\Delta$ on $\gamma$ such that $S\left(Z_{j}\right)=Y_{j}, j=\overline{1, N}$; see [4].

The Poisson formula

$$
P(Z):=\frac{1}{2 \pi} \int_{0}^{2 \pi} S\left(e^{i \theta}\right) \operatorname{Re}\left(\frac{e^{i \theta}+Z}{e^{i \theta}-Z}\right) d \theta, \quad Z \in U,
$$

defines a function $P(Z)$ on $U$, where $S(\zeta)$ is a complex spline function on $\gamma$. We then call $P(Z)$ the complex harmonic spline (C.H.S.) on $U$.

Now we use the C.H.S. to approximate a function belonging to $H(U) \cap C(\bar{U})$.

THEOREM 1. Let $F(Z)$ be a complex harmonic function on $U$, continuous in the closed disc $\bar{U}$. Let $P(Z)$ be the complex harmonic spline defined by $(1)$, where $S(\zeta)$ is the complex cubic spline interpolating at $Z_{j}, S\left(Z_{j}\right)=F\left(Z_{j}\right), j=1, \ldots, N$. Then, $P(Z)$ converges uniformly to $F(Z)$ in the closed disc $\bar{U}$. Moreover, we obtain the error estimation

$$
|F(Z)-P(Z)|<K(R) \omega(F,|\Delta|), \quad Z \in U
$$

where

$$
\begin{aligned}
K(R) & =\operatorname{Min}\left(5.13 R+7.13,0.07 R^{2}+1.5\right), \\
R & =\underset{j}{\operatorname{Max}}\left|Z_{j+1}-Z_{j}\right| / \operatorname{Min}_{j}\left|Z_{j+1}-Z_{j}\right|
\end{aligned}
$$

and $\omega$ is the modulus of continuity of $F$ on $\gamma,|\Delta|=\operatorname{Max}_{j}\left|Z_{j+1}-Z_{j}\right|$.

Proof. Since the error function $E(Z)=F(Z)-P(Z)$ belongs to $H(U)$, by Schwarz's theorem and the condition on $F$ we conclude that $E(Z)$ is continuous in $\bar{U}$. If we can estimate $E(Z)$ on $\gamma$, then, by the maximum modulus principle, (2) follows.

Now we prove the following

LEMMA 1. Let $Y(Z)$ be continuous on $\gamma$, and $S(Z)$ be the complex cubic spline with nodes $\Delta$ such that $S\left(Z_{j}\right)=Y\left(Z_{j}\right)(j=\overline{1, N})$. Then we have

$$
|S(Z)-Y(Z)|<K(R) \omega(Y,|\Delta|), \quad Z \in \gamma,
$$

where $R$ is the mesh ratio defined in Theorem 1 , and $\omega(Y,|\Delta|)$ is the modulus of continuity of $Y$ on $\gamma, K(R)$ being defined as in (2).

Proof. For $Z$ on $\gamma_{j-1}\left(\gamma_{j-1}=\widehat{Z_{j-1} Z_{j}}\right)$

$$
\begin{aligned}
S(Z)= & \frac{m_{j-1}}{h_{j-1}^{2}}\left(Z_{j}-Z\right)^{2}\left(Z-Z_{j-1}\right)-\frac{m_{j}}{h_{j-1}^{2}}\left(Z-Z_{j-1}\right)^{2}\left(Z_{j}-Z\right) \\
& +\frac{Y_{j-1}}{h_{j-1}^{3}}\left(Z_{j}-Z\right)^{2}\left[2\left(Z-Z_{j-1}\right)+h_{j-1}\right] \\
& +\frac{Y_{j}}{h_{j-1}^{3}}\left(Z-Z_{j-1}\right)^{2}\left[2\left(Z_{j}-Z\right)+h_{j-1}\right],
\end{aligned}
$$

where $h_{j-1}=Z_{j}-Z_{j-1}, Y_{j}=S\left(Z_{j}\right), m_{j}=S^{\prime}\left(Z_{j}\right)$. 
The quantities $m_{j}$ satisfy the requirement that $S^{(2)}\left(Z_{j}-\right)=S^{(2)}\left(Z_{j}+\right)$ for $j=\overline{1, N}$ :

$$
a_{j} m_{j-1}+2 m_{j}+b_{j} m_{j+1}=Q_{j}
$$

where

$$
a_{j}=\frac{h_{j}}{h_{j-1}+h_{j}}, \quad b_{j}=1-a_{j}, \quad Q_{j}=3 a_{j} \frac{Y_{j}-Y_{j-1}}{h_{j-1}}+3 b_{j} \frac{Y_{j+1}-Y_{j}}{h_{j}} .
$$

(5) may be written in matrix form as

(6)

$$
A m=Q .
$$

Since we can prove the following inequalities:

(7)

$$
\begin{aligned}
\left\|A^{-1}\right\| \leqslant \frac{1}{\min _{j}\left(2-\left|a_{j}\right|-\left|b_{j}\right|\right)} \leqslant \frac{1}{2-\sqrt{2}}, \\
\lambda_{j}(Z)=\frac{\left|\left(Z-Z_{j-1}\right)\left(Z-Z_{j}\right)^{2}\right|}{\left|h_{j-1}\right|^{2}} \leqslant \frac{\left|h_{j-1}\right|}{2} \text { for } Z \in \gamma_{j-1}, \\
\mu_{j}(Z)=\frac{\left|\left(Z-Z_{j-1}\right)^{2}\left(Z-Z_{j}\right)\right|}{\left|h_{j-1}\right|^{2}} \leqslant \frac{\left|h_{j-1}\right|}{2} \text { for } Z \in \gamma_{j-1}, \\
g(\lambda)=\left|\frac{3 \lambda}{2 h_{j-1}}-\frac{2 \lambda^{3}}{h_{j-1}^{3}}\right| \leqslant 1, \quad \lambda=Z-\frac{1}{2}\left(Z_{j-1}+Z_{j}\right), Z \in \gamma_{j-1},
\end{aligned}
$$

it follows from (6) and (7) that

$$
\left|m_{j}\right| \leqslant \max _{k}\left|Q_{k}\right| \sum_{l=1}^{N}\left|A_{j l}^{-1}\right| \leqslant \frac{\max _{k}\left|Q_{k}\right|}{2-\sqrt{2}} .
$$

For $Z \in \gamma_{k-1}$, in view of (4), (7), (8) we have

$$
\text { (9) } \begin{aligned}
|S(Z)-Y(Z)| \leqslant & \max _{k}\left|m_{k}\right|\left(\lambda_{k}(Z)+\mu_{k}(Z)\right)+g(\lambda)\left|Y_{k}-Y_{k-1}\right| \\
& +\left|\frac{Y_{k}+Y_{k-1}}{2}-Y(Z)\right| \\
& \leqslant \frac{\max _{k}\left|Q_{k}\right|}{2-\sqrt{2}}\left|h_{k-1}\right|+\left|\frac{Y_{k}+Y_{k-1}}{2}-Y(Z)\right|+\left|Y_{k}-Y_{k-1}\right| .
\end{aligned}
$$

From (9) we can easily prove the following inequality

$$
|S(Z)-Y(Z)| \leqslant(5.13 R+7.13) \omega(Y,|\Delta|) .
$$

On the other hand, the continuity of $S^{\prime}(Z)$ at $Z$ yields the following expression

$$
b_{j} M_{j-1}+2 M_{j}+a_{j} M_{j+1}=P_{j}, \quad j=\overline{1, N},
$$

where

$$
M_{j}=S^{(2)}\left(Z_{j}\right), \quad P_{j}=\frac{1}{6\left(h_{j-1}+h_{j}\right)}\left[\frac{Y_{j+1}-Y_{j}}{h_{j}}-\frac{Y_{j}-Y_{j-1}}{h_{j-1}}\right] .
$$

(11) may be written in matrix form as

$$
B M=P \text {. }
$$


Since we can prove the following inequalities:

$$
\begin{gathered}
\left|\alpha_{j}\right|=\left|\left(Z_{j}-Z\right)\left[\left(Z_{j}-Z\right)^{2}-h_{j-1}^{2}\right]\right|\left|6 h_{j-1}\right|^{-1} \leqslant \frac{\left|h_{j-1}\right|^{2}}{12} \quad \text { for } Z \in \gamma_{j-1}, \\
\left|\beta_{j}\right|=\left|\left(Z-Z_{j-1}\right)\left[\left(Z-Z_{j-1}\right)^{2}-h_{j-1}^{2}\right]\right|\left|6 h_{j-1}\right|^{-1} \leqslant \frac{\left|h_{j-1}\right|^{2}}{12} \quad \text { for } Z \in \gamma_{j-1}, \\
\left|P_{j}\right| \leqslant \frac{\omega(Y,|\Delta|)}{3 \sqrt{2}\left|h_{j} h_{j-1}\right|},
\end{gathered}
$$

it follows from (12) and (13) that

$$
\left|M_{j}\right| \leqslant \frac{(1+\sqrt{2}) \omega(Y,|\Delta|)}{6 \operatorname{Min}_{k}\left|h_{k} h_{k-1}\right|}, \quad j=1, \ldots, N .
$$

For $Z \in \gamma_{j}$, in view of (13), (14), we have

$$
\begin{aligned}
|S(Z)-Y(Z)| & =\left|\alpha_{j} M_{j-1}+\beta_{j} M_{j}+\left(\frac{Y_{j}+Y_{j-1}}{2}-Y(Z)\right)\right| \\
& -\left(Y_{j}-Y_{j-1}\right) \frac{Z_{j}+Z_{j-1}-2 Z}{2 h_{j-1}} \mid \\
& \leqslant\left(\frac{3}{2}+\frac{1+\sqrt{2}}{36} R^{2}\right) \omega(Y,|\Delta|)<\left(1.5+0.07 \dot{R}^{2}\right) \omega(Y,|\Delta|) .
\end{aligned}
$$

(3) is obtained from (10) and (15).

Remark 1. A similar estimate for the real periodic cubic splines and functions $f \in C(-\infty, \infty)$ has been obtained by Sharma and Meir [11], where they have an error $O\left(R^{2}\right) \omega(\delta)$.

From Lemma 1, the proof of Theorem 1 is complete.

We note that the coefficient $K(R)$ in the error estimation (2), (3) is a function of $R$. If $R=1$ or $R>1$ but not too large, ${ }^{*}$ then $K(R)=1.5+0.07 R^{2}$; if $R$ is sufficiently large, then $K(R)=5.13 R+7.13$.

Remark 2. From Theorem 1, we see that if $|\Delta| \rightarrow 0$, and $R$ is bounded, then $P(Z)$ converges uniformly to $F(Z)$ on the closed disc $\bar{U}$.

In particular, if $F(Z)$ is analytic in $U$ and continuous in $\bar{U}$, then the approximation C.H.S. $P(Z)$ converges uniformly to $F(Z)$ on the closed $\bar{U}$.

Let $\zeta=\zeta(t)(0 \leqslant t<2 \pi)$ be the parametric representation of $\Gamma$. Suppose $0<$ $\left|\zeta^{\prime}(t)\right|<\infty$. Then $Y_{j}=F\left(Z_{j}\right)(j=\overline{1, N})$ may be obtained by solving the integral equations as in [9], [10], and then the cubic spline $S(Z)$ which interpolates $F(Z)$ at $Z_{j}(j=\overline{1, N})$ may be easily constructed [4].

The closed Jordan curve $\Gamma$ is said to satisfy the Ljapunov condition (see $[8, \mathrm{p}$. 122]), if it can be represented by $\zeta=\zeta(s), 0 \leqslant s<L$, where $L$ is the length of $\Gamma$ and $s$ the arc length of $\Gamma$, measured from a fixed point, such that if $s$ varies from 0 to $L$,

\footnotetext{
* In fact, if $R>R_{1}(=74.40)$, then $K(R)=5.13 R+7.13$; if $1 \leqslant R \leqslant R_{1}$, then $K(R)=0.07 R^{2}+1.5$, where $R_{1}$ is the positive root of the equation $7 R^{2}-513 R-563=0$.
} 
then $\zeta(s)$ makes one turn on $\Gamma$ in the positive sense, $\Gamma$ has a tangent at every point, which varies continuously, and $\zeta^{\prime}(s)$ satisfies the following Hölder condition:

$$
\left|\zeta^{\prime}\left(s_{1}\right)-\zeta^{\prime}\left(s_{2}\right)\right| \leqslant J\left|s_{1}-s_{2}\right|^{\alpha} . \quad 0<\alpha \leqslant 1, J=\text { const. }
$$

If the function $W=F(Z)$ maps $U$ conformally onto $D$ and $\Gamma=\partial D$ satisfies the Ljapunov condition, then $F^{\prime}(Z)$ exists in $U$, is different from zero, and satisfies the same Hölder condition:

$$
\left|F^{\prime}\left(e^{i \theta_{1}}\right)-F^{\prime}\left(e^{i \theta_{2}}\right)\right| \leqslant K\left|\theta_{1}-\theta_{2}\right|^{\alpha}, \quad k=\mathrm{const}
$$

(see [8], [12]).

It follows that $F^{\prime}(Z)$ is continuous in $\bar{U}[8$, p. 122].

LEMMA 2. If $\Gamma$ satisfies the Ljapunov condition, and $W=F(Z)$ maps $U$ conformally onto $D, \partial D=\Gamma$, and $S(Z)$ is the complex cubic spline which interpolates $F(Z)$ at $\Delta=\left\{Z_{j}\right\}_{1}^{N}$ - the knots of $S(Z)$-then, for $Z \in \gamma, \gamma=\partial U$, we have

$$
\begin{gathered}
|S(Z)-F(Z)| \leqslant K_{1}|\Delta|^{1+\alpha}, \quad Z \in \gamma, \\
\left|S^{\prime}(Z)-F^{\prime}(Z)\right| \leqslant K_{2}|\Delta|^{\alpha}, \quad Z \in \gamma, \\
\left|\frac{\left(S^{\prime}\left(t_{1}\right)-F^{\prime}\left(t_{1}\right)\right)-\left(S^{\prime}\left(t_{2}\right)-F^{\prime}\left(T_{2}\right)\right)}{|\Delta|^{\alpha-\delta}}\right| \leqslant K_{3}\left|t_{1}-t_{2}\right|^{\delta}, \quad t_{1}, t_{2} \in \gamma .
\end{gathered}
$$

for any $\delta, 0<\delta<\alpha$, where $R$ is the mesh ratio and

$$
\begin{aligned}
& |\Delta|=\max _{1}\left|Z_{j}-Z_{j \cdots 1}\right|, \\
& K_{1}=\frac{\pi}{2} K_{2}, \quad K_{2}=(26+14 \sqrt{2}) \frac{K}{\alpha+1}\left(\frac{\pi}{2}\right)^{\alpha+1} . \\
& K_{3}=2\left(\frac{38+40 \sqrt{2}}{\alpha+1} \frac{\pi}{2}+1\right)\left(\frac{\pi}{2}\right)^{\alpha} K R .
\end{aligned}
$$

Proof. From (5),

$$
A\left(m-\frac{1}{3} Q\right)=(3 I-A) \frac{1}{3} Q .
$$

The $j$ th element of the vector $(3 I-A) \frac{1}{3} Q$ may be estimated as follows. Since

$$
\left|\frac{1}{h_{j}} \int_{Z_{1}}^{Z_{1} \cdot 1}\left(F^{\prime}(t)-F^{\prime}\left(Z_{j+1}\right)\right) d t\right| \leqslant \frac{K}{\alpha+1}\left(\frac{\pi}{2}\right)^{\alpha+1}|\Delta|^{\alpha},
$$

we have

$$
\begin{aligned}
& {[(3 I-A)-Q],=\left|\frac{-a_{j} Q_{j-1}}{3}+\frac{Q_{j}}{3}-\frac{b_{j} Q_{j+1}}{3}\right|} \\
& \quad \leqslant 4 \max _{k} \mid \frac{1}{h_{k}} \int_{Z_{h}}^{Z_{k}+1}\left(F^{\prime}(t)-\left.F^{\prime}\left(Z_{k+1}\right) d t\left|\leqslant \frac{4 K}{\alpha+1}\left(\frac{\pi}{2}\right)^{\alpha+1}\right| \Delta\right|^{\alpha} .\right.
\end{aligned}
$$

From (19), we have

$$
\begin{aligned}
\mid m_{j}-\left(b_{j} \frac{F_{j+1}-F_{j}}{h_{j}}+\right. & \left.a_{j} \frac{F_{j}-F_{j-1}}{h_{j-1}}\right) \mid \\
& \leqslant\left\|A^{-1}\right\| \max _{k}\left[(3 I-A) \frac{1}{3} Q\right]_{k}\left(\frac{4 K(\pi / 2)^{\alpha+1}|\Delta|^{\alpha}}{(\alpha+1)(2-\sqrt{2})}\right) .
\end{aligned}
$$


Therefore, from (20), (22) and $a_{j}+b_{j}=1$, we find

$$
\begin{aligned}
\left|m_{j}-\frac{F_{j}-F_{j-1}}{h_{j-1}}\right| \leqslant\left|m_{j}-\left(b_{j} \frac{F_{j+1}-F_{j}}{h_{j}}+a_{j} \frac{F_{j}-F_{j-1}}{h_{j-1}}\right)\right| \\
+\mid \frac{1}{h_{j}} \int_{Z_{j}}^{Z_{j+1}}\left(F^{\prime}(t)-F^{\prime}\left(Z_{j}\right)\right) d t \\
\quad-\frac{1}{h_{j-1}} \int_{Z_{j-1}}^{Z_{j}}\left(F^{\prime}(t)-F^{\prime}\left(Z_{j}\right)\right) d t \mid \\
\leqslant\left(\frac{2}{2-\sqrt{2}}+1\right) \frac{2 K}{\alpha+1}\left(\frac{\pi}{2}\right)^{\alpha+1}|\Delta|^{\alpha} .
\end{aligned}
$$

Furthermore,

$$
\begin{aligned}
\left|S^{\prime}(Z)-\frac{F_{j}-F_{j-1}}{h_{j-1}}\right| \leqslant\left|\frac{3 \varepsilon^{2}}{h_{j-1}^{2}}-\frac{1}{4}\right|\left|m_{j-1}+m_{j}-2 \frac{F_{j}-F_{j-1}}{h_{j-1}}\right| \\
+\left|\frac{\varepsilon}{h_{j-1}}\right|\left|m_{j}-m_{j-1}\right| \text { for } Z \in \gamma_{j-1},
\end{aligned}
$$

where

$$
\varepsilon=\frac{Z_{j}+Z_{j-1}}{2}-Z_{j}=\tilde{Z}-Z_{j}
$$

and evidently,

$$
\varepsilon \leqslant \frac{\left|h_{j-1}\right|}{\sqrt{2}}, \quad\left|\frac{3 \varepsilon^{2}}{h_{j-1}^{2}}-\frac{1}{4}\right| \leqslant \frac{7}{4} .
$$

Let

$$
Y_{j}=\frac{1}{h_{j}} \int_{Z_{j}}^{Z_{j+1}}\left(F^{\prime}(t)-F^{\prime}\left(Z_{j+1}\right)\right) d t
$$

Then, from (20), (22),

$$
\text { (27) } \begin{aligned}
\left|m_{j}-m_{j-1}\right| & \leqslant\left|m_{j}-\frac{F_{j}-F_{j-1}}{h_{j-1}}-\left(m_{j-1}-\frac{F_{j-1}-F_{j-2}}{h_{j-2}}\right)\right|+\left|Y_{j-1}-Y_{j-2}\right| \\
& \leqslant\left(\frac{4}{2-\sqrt{2}}+3\right) \times \frac{2 K}{\alpha+1}\left(\frac{\pi}{2}\right)^{\alpha+1}|\Delta|^{\alpha} .
\end{aligned}
$$

Combining (24)-(27), we obtain

$$
\left|S^{\prime}(Z)-\frac{F_{j}-F_{j-1}}{h_{j-1}}\right| \leqslant(25+14 \sqrt{2}) \frac{K}{\alpha+1}\left(\frac{\pi}{2}\right)^{\alpha+1}|\Delta|^{\alpha}, \quad Z \in \gamma_{j-1} .
$$

Thus, for $Z \in \gamma_{j-1}$,

$$
\begin{aligned}
\left|S^{\prime}(Z)-F^{\prime}(Z)\right| & \left.\leqslant\left|S^{\prime}(Z)-\frac{F_{j}-F_{j-1}}{h_{j-1}}\right|+\mid \frac{1}{h_{j-1}} \int_{Z_{j-1}}^{Z_{j}} F^{\prime}(Z)-F^{\prime}(t)\right) d t \mid \\
& \leqslant(26+14 \sqrt{2}) \frac{K}{\alpha+1}\left(\frac{\pi}{2}\right)^{\alpha+1}|\Delta|^{\alpha} .
\end{aligned}
$$


Inequality $(17)$ is proved.

Inequality (16) follows from (17) by an integration. Since

$$
\begin{aligned}
& \left|S^{\prime}(Z)-S^{\prime}(t)\right|=|Z-t|\left\lfloor\frac{m_{j-1}-\frac{F_{j}-F_{j-1}}{h_{j-1}}}{h_{j-1}}+\frac{m_{j}-\frac{F_{j}-F_{j-1}}{h_{j-1}}}{h_{j-1}}\right] \\
& \left.\times\left(\frac{3(Z+t)-6 \tilde{Z}}{h_{j-1}}\right)+\frac{m_{j}-m_{j-1}}{h_{j-1}}\right) \text {, } \\
& \tilde{Z}=\frac{1}{2}\left(Z_{j-1}+Z_{j}\right), t, Z \in \gamma_{j-1},
\end{aligned}
$$

from (23), (27), (29) and $\left|(3(Z+t)-6 \dot{Z}) / h_{j-1}\right| \leqslant 3 \sqrt{2}$, we have

$$
\left|S^{\prime}(Z)-S^{\prime}(t)\right| \leqslant \frac{2(19+20 \sqrt{2})}{\alpha+1}\left(\frac{\pi}{2}\right)^{\alpha+1} K|\Delta|^{\alpha}\left|\frac{Z-t}{h_{j-1}}\right| .
$$

Therefore, if $Z, t \in \gamma_{j-1}$, we have

$$
\begin{aligned}
& \frac{\left|\left(S^{\prime}(Z)-F^{\prime}(Z)\right)-\left(S^{\prime}(t)-F^{\prime}(t)\right)\right|}{|\Delta|^{\alpha-\delta}} \\
& \leqslant\left[\frac{19+20 \sqrt{2}}{\alpha+1} \pi R+1\right]\left(\frac{\pi}{2}\right)^{\alpha} K|Z-t|^{\delta},
\end{aligned}
$$

where $R=|\Delta| / \min _{j}\left|Z_{j}-Z_{j-1}\right|$.

If, on the other hand, $Z \in \gamma_{j}, t \in \gamma_{l}, j \neq l$, we distinguish three cases:

(1) If $|t-Z| \geqslant|\Delta|$, then from (17)

$$
\frac{\left|\left(S^{\prime}(Z)-F^{\prime}(Z)\right)-\left(S^{\prime}(t)-F^{\prime}(t)\right)\right|}{|\Delta|^{\alpha-\delta}} \leqslant 2 K_{2}|\Delta|^{\delta} \leqslant K_{3}|t-Z|^{\delta} .
$$

(2) If $|t-Z|<|\Delta|,|Z-t| \geqslant \min _{j}\left|h_{j}\right|$, then from (17)

$$
\frac{\mid\left(S^{\prime}(Z)-F^{\prime}(Z)\right)-\left(S^{\prime}(t)-F^{\prime}(t)\right)}{|\Delta|^{\alpha-\delta}} \leqslant 2 K_{2}|\Delta|^{\delta} \leqslant K_{3}|t-Z|^{\delta} .
$$

(3) If $|t-Z|<|\Delta|$ and $|Z-t|<\min \left|h_{j}\right|$, we assume, without loss of generality, that $t \in \gamma_{j+1}, Z \in \gamma_{j}$. Then

$$
\begin{aligned}
& \frac{\left|\left(S^{\prime}(Z)-F^{\prime}(Z)\right)-\left(S^{\prime}(t)-F^{\prime}(t)\right)\right|}{|\Delta|^{\alpha-\delta}} \\
& \leqslant \frac{\left|\left(S^{\prime}(Z)-F^{\prime}(Z)\right)-\left(S^{\prime}\left(Z_{j+1}\right)-F^{\prime}\left(A_{j+1}\right)\right)\right|}{|\Delta|^{\alpha-\delta}}, \\
& \frac{\left|\left(S^{\prime}(t)-F^{\prime}(t)\right)-\left(S^{\prime}\left(Z_{j+1}\right)-F^{\prime}\left(Z_{j+1}\right)\right)\right|}{|\Delta|^{\alpha-\delta}} \\
& \leqslant K^{\prime}\left(\left|Z-Z_{j+1}\right|^{\delta}+\left|t-Z_{j+1}\right|^{\delta}\right) \leqslant 2 K^{\prime}|Z-t|^{\delta} \leqslant K_{3}|Z-t|^{\delta},
\end{aligned}
$$

where

$$
K^{\prime}=\left[\frac{19+20 \sqrt{2}}{\alpha+1} \pi R+1\right]\left(\frac{\pi}{2}\right)^{\alpha} K
$$


the second inequality being obtained from (31).

From (31)-(34), formula (18) follows. Q.E.D.

Remark 3. Ahlberg, Nilson and Walsh in [1] estimated the order of errors. Here we estimate not only the order of the errors but also give the explicit form of the error coefficients.

TheOREM 2. Let $\Gamma$ satisfy the Ljapunov condition. Let $W=F(Z)$ be the mapping function from $U$ onto $D, S(Z)$ the complex cubic spline which interpolates $F(Z)$ at $\Delta=\left\{Z_{j}\right\}_{j=1}^{N}$, and $P(Z)$ the C.H.S. with the boundary function $S(Z)$.

Then, for all $Z \in \bar{U}$, we have

$$
\begin{aligned}
|P(Z)-F(Z)| & \leqslant K_{1}|\Delta|^{1+\alpha}, \\
\left|P_{Z}(Z)-F^{\prime}(Z)\right| & \leqslant \xi|\Delta|^{\alpha / 2}, \\
\left|P_{Z}(Z)\right| & \leqslant \xi|\Delta|^{\alpha / 2},
\end{aligned}
$$

where $\xi=\left(2^{\alpha / 2} / \alpha\right) K_{3}+K_{2}|\Delta|^{\alpha / 2}, P_{Z}, P_{\bar{Z}}$ are complex derivatives (see [3]). $K_{1}, K_{2}$, $K_{3}$ are constants as in Lemma 2.

Proof. (35) is obtained directly from the maximum modulus principle and (16). Now we prove (36).

Let $\varphi\left(\zeta, Z_{0}\right)$ represent the function $\left(S^{\prime}(\zeta)-F^{\prime}(\zeta)\right)-\left(S^{\prime}\left(Z_{0}\right)-F^{\prime}\left(Z_{0}\right)\right)$; from (18),

$$
\left|\varphi\left(\zeta, Z_{0}\right)\right| \leqslant K_{3}|\Delta|^{\alpha-\delta}\left|\zeta-Z_{0}\right|^{\delta}
$$

We have [3]

$$
\left|P_{Z}(Z)-F^{\prime}(Z)\right| \leqslant \operatorname{Sup}_{Z_{0} \in \gamma}\left\{\left|\frac{1}{2 \pi} \int_{\gamma} \frac{\varphi\left(\zeta, Z_{0}\right)}{\zeta-Z_{0}} d \zeta+S^{\prime}(Z)-F^{\prime}(Z)\right|\right\}
$$

Since

$$
\int_{\gamma}\left|\zeta-Z_{0}\right|^{\delta-1}|d \zeta| \leqslant 2^{\delta} \pi / \delta
$$

let $\delta=\alpha / 2$; then

$$
\left|\frac{1}{2 \pi} \int_{\gamma} \frac{\varphi\left(\zeta, Z_{0}\right)}{\zeta-Z_{0}} d \zeta\right| \leqslant \frac{2^{\alpha / 2}}{\alpha} K_{3}|\Delta|^{\alpha / 2} .
$$

From (17), (39) and (38), for $Z \in \bar{U}$ we have

$$
\left|P_{Z}(Z)-F^{\prime}(Z)\right| \leqslant\left[\frac{2^{\alpha / 2}}{\alpha} K_{3}+K_{2}|\Delta|^{\alpha / 2}\right]|\Delta|^{\alpha / 2}=\xi|\Delta|^{\alpha / 2} .
$$

(36) is proved.

For $Z \in \bar{U}$, from (18) and (39), we have [3]

$$
\begin{aligned}
& \left|P_{\bar{Z}}(Z)\right| \leqslant \frac{1}{2 \pi} \operatorname{Sup}_{Z_{0} \in \gamma}\left|\int_{\gamma} \frac{\left(\zeta S^{\prime}(\zeta)-Z_{0} S^{\prime}\left(Z_{0}\right)\right)-\left(\zeta F^{\prime}(\zeta)-Z_{0} F^{\prime}\left(Z_{0}\right)\right)}{\zeta-Z_{0}} d \zeta\right| \\
& \quad \leqslant \frac{1}{2 \pi} \operatorname{Sup}_{Z_{0} \in \gamma}\left|\int_{\gamma} \frac{\zeta\left[\left(S^{\prime}(\zeta)-F^{\prime}(\zeta)\right)-\left(S^{\prime}\left(Z_{0}\right)-F^{\prime}\left(Z_{0}\right)\right)\right]+\left(\zeta-Z_{0}\right)\left(S^{\prime}\left(Z_{0}\right)-F^{\prime}\left(Z_{0}\right)\right)}{\zeta-Z_{0}} d \zeta\right| \\
& \quad \leqslant \frac{1}{2 \pi}\left\{K_{3}|\Delta|^{\alpha-\delta} 2^{\delta} \delta^{-1}+2 \pi K_{2}|\Delta|^{\alpha}\right\} .
\end{aligned}
$$


Let $\delta=\alpha / 2$; then

$$
\left|P_{\bar{Z}}(Z)\right| \leqslant \xi|\Delta|^{\alpha / 2} \quad \text { for } Z \in \bar{U} \text {. }
$$

(37) is thus proved.

THEOREM 3. If $\Gamma$ satisfies the Ljapunov condition and $P(Z)$ is the complex harmonic spline defined in Theorem 2, and if

$$
|\Delta|<\frac{1}{2 K_{2}}\left\{\left[2^{\alpha} \alpha^{-2} K_{3}^{2}+2 K_{2} \cdot \min _{\zeta \in \gamma}\left|F^{\prime}(\zeta)\right|\right]^{1 / 2}-2^{\alpha / 2} \alpha^{-1} K_{3}\right\},
$$

then $P(Z)$ is an open mapping on $U$.

Proof. The Jacobian $J(Z)$ does not vanish, since from (43),

$$
J(Z)=\left|P_{Z}(Z)\right|^{2}-\left|P_{\bar{Z}}(Z)\right|^{2} \geqslant\left|F^{\prime}(Z)\right|\left(\left|F^{\prime}(Z)\right|-2 \xi|\Delta|^{\alpha / 2}\right)>0
$$

for all $Z \in U$, thus $P(Z)$ is an open mapping. Q.E.D.

THEOREM 4. If $\Gamma$ satisfies the Ljapunov condition and $P(Z)$ is the complex harmonic spline defined in Theorem 2 and if

$$
|\Delta|<\frac{1}{2 K_{2}}\left\{\left[2^{\alpha} \alpha^{-2} K_{3}^{2}+2 K_{2} m_{F}\right]^{1 / 2}-2^{\alpha / 2} \alpha^{-1} K_{3}\right\}
$$

where

$$
m_{F}=\inf _{Z_{1}, Z_{2} \in \gamma}\left|\frac{F\left(Z_{1}\right)-F\left(Z_{2}\right)}{Z_{1}-Z_{2}}\right|,
$$

then $W=P(Z)$ is a homeomorphism.

Proof. From Kellogg's theorem [12, pp. 361-364], it is easy to see that $m_{F}>0$.

Define

$$
H\left(Z_{1}, Z_{2}\right)=\frac{P\left(Z_{2}\right)-P\left(Z_{1}\right)}{Z_{2}-Z_{1}}, \quad F\left(Z_{1}, Z_{2}\right)=\frac{F\left(Z_{2}\right)-F\left(Z_{1}\right)}{Z_{2}-Z_{1}}
$$

Then

$$
\begin{aligned}
&\left|H\left(Z_{1}, Z_{2}\right)-F\left(Z_{1}, Z_{2}\right)\right| \leqslant \frac{1}{\left|Z_{2}-Z_{1}\right|} \int_{0}^{l}\left|\frac{\partial P}{\partial l_{\theta}}-\frac{\partial F}{\partial l_{\theta}}\right| d l \\
& \quad=\frac{1}{\left|Z_{2}-Z_{1}\right|} \int_{0}^{l}\left|\left(P_{Z}-F^{\prime}\right) e^{i \theta}+\left(P_{\bar{Z}}-0\right) e^{-i \theta}\right| d l \\
& \leqslant \frac{1}{\left|Z_{2}-Z_{1}\right|}\left\{\operatorname{Sup}_{Z \in \gamma}\left|P_{Z}(Z)-F^{\prime}(Z)\right|+\operatorname{Sup}_{Z \in \gamma}\left|P_{\bar{Z}}(Z)\right|\right\} \int_{0}^{l} d l \\
& \leqslant 2 \xi|\Delta|^{\alpha / 2},
\end{aligned}
$$

where $l=\left|Z_{2}-Z_{1}\right|, \partial P / \partial l_{\theta}, \partial F / \partial l_{\theta}$ are the directional derivatives along the straight line joining $Z_{1}$ and $Z_{2}$, and $\theta$ is the inclination of the line $\overline{Z_{1} Z_{2}}$.

It follows from (45) that

$$
\left(m_{F}-2 \xi|\Delta|^{\alpha / 2}\right)\left|Z_{2}-Z_{1}\right| \leqslant\left|P\left(Z_{2}\right)-P\left(Z_{1}\right)\right| \leqslant\left(M_{F}+2 \xi|\Delta|^{\alpha / 2}\right)\left|Z_{2}-Z_{1}\right| .
$$

(46), (44) lead to the following conclusion: $P\left(Z_{2}\right)=P\left(Z_{1}\right)$ if and only if $Z_{1}=Z_{2}$. Q.E.D. 
Following the proof given in [3] we conclude that if $\Gamma$ and $P(Z), F(Z)$ are defined as in Theorem 2 , then $P(Z)$ maps $U$ onto a simply connected domain $D_{p}$. The mapping is one-to-one. Moreover,

$$
\operatorname{Lim}_{|\Delta| \rightarrow 0} D_{p}=D, \quad D \text { is the interior of } \Gamma,
$$

in Carathéodory's sense.

3. The Calculation of C.H.S. and Examples. We rewrite the C.H.S. as follows

$$
P(Z)=P_{1}(Z)+P_{2}(\hat{Z})
$$

where

$$
P_{1}(Z)=\frac{1}{2 \pi i} \int_{\gamma} \frac{S(\zeta)}{\zeta-Z} d \zeta, \quad P_{2}(\hat{Z})=\frac{-1}{2 \pi i} \int_{\gamma} \frac{S(\zeta)}{\zeta-\hat{Z}} d \zeta, \quad \hat{Z}=(\bar{Z})^{-1},
$$

$Z \in \bar{U}, \gamma=\partial U . S(\zeta)$ is the interpolating cubic spline.

Suppose that $S(\zeta)=S_{j}(\zeta), \zeta \in \gamma_{j}, \gamma_{j}=\overparen{Z_{j} Z_{j+1}}$; then (see [2], [3]) we have

$$
\begin{aligned}
P_{1}(Z) & =\frac{1}{2 \pi i} \sum_{j=1}^{N} S_{j}(Z) \int_{Z_{j}}^{Z_{j+1}} \frac{d \zeta}{\zeta-Z} \\
& =\frac{1}{2 \pi i} \sum_{j=1}^{N} S_{j}(Z)\left(\operatorname{Ln}\left|\frac{Z_{j+1}-Z}{Z_{j}-Z}\right|+i \theta_{j, j+1}(Z)\right), \\
P_{2}(\hat{Z}) & =\frac{-1}{2 \pi i} \sum_{j=1}^{N} S_{j}(\hat{Z})\left(\operatorname{Ln}\left|\frac{Z_{j+1}-\hat{Z}}{Z_{j}-\hat{Z}}\right|+i \varphi_{j, j+1}(\hat{Z})\right),
\end{aligned}
$$

where

$$
\begin{aligned}
& \theta_{j, j+1}(Z)=\int_{Z_{j}}^{Z_{j+1}} d \arg (\zeta-Z), \\
& \varphi_{j, j+1}(Z)=\int_{Z_{j}}^{Z_{j+1}} d \arg (\zeta-Z) .
\end{aligned}
$$

Both functions $\theta_{j, j+1}(Z), \varphi_{j, j+1}(Z)$ are single valued and continuous. They may be calculated as follows.

Denote by $l_{j, j+1}$ the straight line joining the points $Z_{j}$ and $Z_{j+1}$; the $Z$-plane $E_{2}$ is divided by $l_{j, j+1}$ into $\Omega_{1}$ and $\Omega_{2}$ say, the half-plane $\Omega_{2}$ contains the circular arc $\gamma_{j}$ (we have stipulated that $\left.\left|\gamma_{j}\right| \leqslant \pi\right)$.

Then $\theta_{j, j+1}, \varphi_{j, j+1}$ may be represented as follows:

$$
\theta_{j, j+1}(Z)= \begin{cases}\lambda, & Z \in U \cap \Omega_{1}, \\ \pi, & Z \in U \cap l_{j, j+1}, \\ 2 \pi-\lambda, & Z \in U \cap \Omega_{2},\end{cases}
$$

where

$$
\begin{gathered}
\lambda=\cos ^{-1}\left(\frac{\left(X_{j}-X\right)\left(X_{j+1}-X\right)+\left(Y_{j}-Y\right)\left(Y_{j+1}-Y\right)}{\left(\left(X_{j}-X\right)^{2}+\left(Y_{j}-Y\right)^{2}\right)^{1 / 2}\left(\left(X_{j+1}-X\right)^{2}+\left(Y_{j+1}-Y\right)^{2}\right)^{1 / 2}}\right), \\
Z_{j}=X_{j}+i Y_{j}, \quad Z_{j+1}=X_{j+1}+i Y_{j+1}, \quad Z=X+i Y .
\end{gathered}
$$




$$
\varphi_{j, j+1}(\hat{Z})= \begin{cases}-\lambda, & \hat{Z} \in C U \cap \Omega_{2}, \\ 0, & \hat{Z} \in C U \cap l_{j, j+1}, \\ \lambda, & \hat{Z} \in C U \cap \Omega_{1},\end{cases}
$$

where $\lambda$ is calculated as in (52) but we use $\hat{Z}=\hat{X}+i \hat{Y}$ instead of $Z=X+i Y$.

Let $\xi$ be a point in $E_{2}$, the straight line joining the point $\xi$ and $Z=0$ is denoted by $l$; let $Z_{0}$ (if it exists) be the intersection of $l$ with $l_{j, j+1}$. Then

$$
\begin{array}{ll}
\xi \in \Omega_{1} & \text { if and only if }|\xi|=|| \xi-Z_{0}|-| Z_{0}||, \text { or } l|| l_{j, j+1}, \\
\xi \in \Omega_{2} & \text { if and only if }|\xi|=\left|Z_{0}\right|+\left|\xi-Z_{0}\right|, \\
\xi \in l_{j, j+1} & \text { if and only if }|\xi|=\left|Z_{0}\right| .
\end{array}
$$

But in the programming, instead of (54), (55), (56) we use

$$
\begin{aligned}
|\xi| & >|| \xi-Z_{0}|-| Z_{0}||-\varepsilon, \\
|\xi| & <\left|Z_{0}\right|+\left|\xi-Z_{0}\right|+\varepsilon, \\
|\xi|-\varepsilon & \leqslant\left|Z_{0}\right| \leqslant|\xi|+\varepsilon,
\end{aligned}
$$

respectively, where $\varepsilon$ is a small number, say,

$$
|\varepsilon| \sim 10^{-8} \text {. }
$$

From the formulas (47)-(59) the values of $P(Z)$ (for $Z \subseteq \bar{U}$ ) may be calculated if the spline function $S(\zeta)$ is given.

Now $S(Z)$ may be written as

$$
\begin{array}{ll}
\text { (60) } S(Z)= \begin{cases}a_{3} Z^{3}+a_{2} Z^{2}+a_{1} Z+a_{0}, & Z \in \gamma_{N}, \\
a_{3} Z^{3}+a_{2} Z^{2}+a_{1} Z+a_{0}+\sum_{j=1}^{k} C_{j}\left(Z-Z_{j}\right)^{3}, & Z \in \gamma_{k},\end{cases} & k=1, \ldots, N,
\end{array}
$$

$$
\sum_{j=1}^{N} C_{j} Z_{j}^{\prime}=0, \quad l=0,1,2,3 .
$$

If $\left\{F\left(Z_{j}\right)\right\}_{j-1}^{N}$ are given, then $S(Z)$ may be obtained by solving the system of equations:

$$
\left\{\begin{array}{l}
S\left(Z_{j}\right)=F\left(Z_{j}\right), \quad j=\overline{1, N}, \\
\sum_{j=1}^{N} C_{j} Z_{j}^{\prime}=0, \quad l=0,1,2,3 .
\end{array}\right.
$$

It was proved in [4] that the system of equations (61) can be solved uniquely for $\left\{a_{j}\right\}_{j=0}^{3}$ and $\left\{C_{j}\right\}_{j=1}^{N}$.

In particular, the data values of a conformal mapping function may be obtained as follows:

Let $W=F(Z)$ be a mapping function, such that $F(0)=0, F(1)=\zeta_{0} \in \Gamma, Z=e^{i \theta}$, $\theta=\arg Z=\arg \left(F^{-1}(\zeta)\right)$. 
From $[9$, p. 32, Theorem 9.1], the following system of equations

$$
\begin{gathered}
g+\int_{\Gamma} \operatorname{Ln}|W-\zeta| \sigma(\zeta)|d \zeta|=\operatorname{Ln}|W|, \quad W \in \Gamma \\
\int_{\Gamma} \sigma(\zeta)|d \zeta|=1
\end{gathered}
$$

has a unique solution $\left(g^{*}, \sigma^{*}\right), g^{*}=0$, and

$$
\theta(\zeta)=2 \pi \int_{\zeta_{0}}^{\zeta} \sigma^{*}(W) d W
$$

Integration is done along $\Gamma$ in the positive direction.

Using the numerical method presented in [10], we have

$$
\theta(W(t))=t+\sum_{j=1}^{n} \frac{\alpha_{j}}{j} \sin j t=-\sum_{j=1}^{n-1} \frac{\beta_{j}}{j} \cos j t+\sum_{j=1}^{n-1} \frac{\beta_{j}}{j},
$$

$0 \leqslant t<2 \pi$, where $\alpha_{j}, \beta_{j}$ are constants.

By solving the equations

$$
\theta(w(t))=\frac{2 \pi l}{N}, \quad l=\overline{1, N},
$$

the data values $F_{l}=F\left(e^{i 2 \pi l / N}\right), l=\overline{1, N}$, are obtained.

We have written a complete program for the whole system.

The input is the parametric representation of the curve $\Gamma$, and the output is the graph of the C.H.S. We give nine examples in [5].

Remark 4 . If $\Gamma$ is an arbitrary rectifiable Jordan curve, under a stronger assumption on the approximated function $F$, we can obtain

$$
\lim _{\max \left|\Delta_{1}\right| \rightarrow 0} \int_{\Gamma} \frac{S_{\Delta}(\zeta) d \zeta}{\zeta-Z}=\int_{\Gamma} \frac{F(\zeta) d \zeta}{\zeta-Z} \quad \text { for all } Z \in \bar{D}
$$

$\bar{D}=(D \cup \Gamma, D=$ interior to $\Gamma)$.

But the limit value on the left side of (65) is more complicated than in the special case where $\Gamma$ is the circle, since in this case, we use $P(Z)$-the complex harmonic spline-in which case the boundary value of $P(Z)$ is $S\left(Z_{0}\right)$.

We shall discuss this in more detail in another paper.

Acknowledgements. We would like to give special thanks to Professor Tore Håvie, who has both written the FFT program and suggested how to use it. Without his help we would have been unable to finish this paper in the very short time that we had at our disposal, as we had virtually no previous experience with the FFT and its use.

We would like to thank the referee and Professor W. Gautschi for their constructive suggestions. 
We would also like to thank Miss A. Kajander who has typed this paper from a sometimes difficult manuscript.

Institute of Mathematics

Academia Sinica

Beijing. China

The University of Trondheim

The Norwegian Institute of Technology

Division of Numerical Mathematics

7934 Trondheim-NTH, Norway

I. J. H. Ahlberg, E. N. Nilson \& J. L. Walsh, “Complex cubic splines,” Trans. Amer. Math. Soc., v. 129. 1967, pp. 391-413.

2. J. H. Ahlberg, Splines in the Complex Plane, Approximations with Special Emphasis on Spline Functions (Proc. Sympos. Univ. Wisconsin, Madison, Wis., 1969), Academic Press, New York, 1969, pp. $1-27$.

3. Chen Han - Lin, Complex Harmonic Splines, Interpolation and Approximation on the Unit Circle, Part II. Tech. Report, Math. and Comp. No. 3/81, ISBN 82-7151-039-8.

4. Chen Han - Lin, “Complex spline functions,” Sci. Sinica, v. 24, No. 2, 1981, pp. 160-169.

5. Chen Han - Lin \& Tron Hvaring, A New Method for the Approximation of Conformal Mapping on the Unit Circle, Tech. Report, Math. and Comp. No. 6/82, ISBN 82-7151-049-5.

6. L. FejÉR, “Über Interpolation,” Göttingen Nachr, 1916, pp. 66-91.

7. D. GaIER, “Integralgleichungen erster Art und konforme Abbildung," Math. Z., v. 47, 1976, pp. $113-129$

8. M. A. LaWrentuew \& B. W. Schabat, Methoden der komplexen Funktionentheorie, VEB Deutscher Verlag der Wissenschaften, Berlin, 1967.

9. L. Reichel, On Polynomial Approximation in the Complex Plane with Application to Conformal Mapping, TRITA-Na-8102, Dept. Comput. Sci., Royal Institute of Technology, Stockholm.

10. L. Reichel, On the Determination of Boundary Collocation Points for Solving some Problems for the Laplace Operator, TRITA-NA-8006, Dept. Comput. Sci., Royal Institute of Technology, Stockholm.

11. A. Sharma \& A. MeIR, "Degree of approximation of spline interpolation," J. Math. and Mech., v. 15. No. 1-6, 1966, pp. 759-767.

12. M. Tsuj, Potential Theory in Modern Function Theory, Maruzen, Tokyo, 1959. 